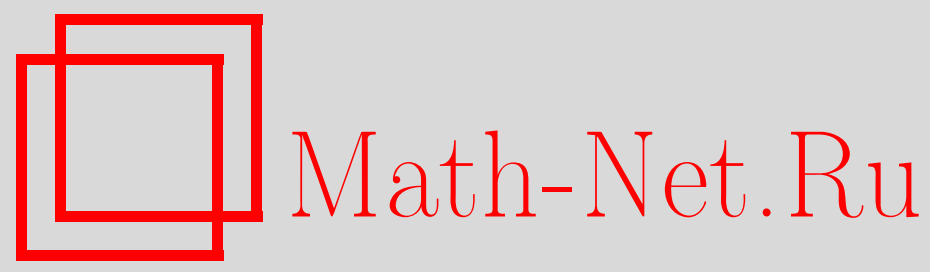

А. В. Ниукканен, Распространение принципа факторизации на гипергеометрические ряды общего вида, Матем. заметкu, 2000, том 67, выпуск 4, 573-581

DOI: https://doi.org/10.4213/mzm872

Использование Общероссийского математического портала Math-Net.Ru подразумевает, что вы прочитали и согласны с пользовательским соглашением http://www.mathnet.ru/rus/agreement

Параметры загрузки:

IP : 54.81 .137 .203

26 апреля 2023 г., $15: 43: 36$

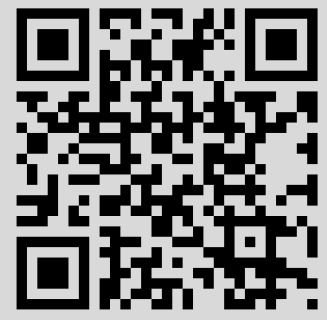




\title{
РАСПРОСТРАНЕНИЕ ПРИНЦИПА ФАКТОРИЗАЦИИ НА ГИПЕРГЕОМЕТРИЧЕСКИЕ РЯДЫ ОБЩЕГО ВИДА
}

\section{А. В. Ниукканен}

\begin{abstract}
Показано, что формулы операторной факторизации гипергеометрических функций, полученные в предыдущих работах автора, допускают распространение на гипергеометрические ряды наиболее общего вида. Такое обобщение не приводит к усложнению технического аппарата метода факторизации. В качестве примера, иллюстрирующего практическую эффективность полученных в работе формул, дан анализ трансформационных свойств горновского ряда $G_{3}$, имеющего типичную для общих гипергеометрических функций структуру. Показано, что формула преобразования Эрдейи, связывающая ряд $G_{3}$ с функцией Аппеля $F_{2}$, содержит ошибочные выражения в аргументах ряда $G_{3}$. Найден правильный аналог формулы Эрдейи и приведены некоторые новые преобразования ряда $G_{3}$.

Библиография: 12 названий.
\end{abstract}

1. Введение. Как показано в [1]-[3], применение метода операторной факторизации значительно облегчает исследование свойств многих гипергеометрических функций. Основная идея метода факторизации состоит во введении простой дифференциальной операции (именуемой далее операцией $\Omega$-умножения) над функциями $u\left(x_{1}, \ldots, x_{N}\right)$, $v\left(x_{1}, \ldots, x_{N}\right)$ :

$$
\left\langle u * v \mid x_{1}, \ldots, x_{N}\right\rangle=\left.u\left(\frac{d}{d s_{1}}, \ldots, \frac{d}{d s_{N}}\right) v\left(x_{1} s_{1}, \ldots, x_{N} s_{N}\right)\right|_{s_{1}=\cdots=s_{N}=0}
$$

позволяющей выражать сложные гипергеометрические ряды через гипергеометрические функции более простого вида и использовать свойства последних для анализа свойств исходных гипергеометрических функций. Обозначим через ${ }^{N} F\left[D ; x_{1}, \ldots, x_{N}\right]$ произвольный степенной ряд от $N$ переменных $x_{1}, \ldots, x_{N}$ с коэффициентами $D(i) \equiv$ $D\left(i_{1}, \ldots, i_{N}\right)$, где $\boldsymbol{i}=\left(i_{1}, \ldots, i_{N}\right)$ - множество переменных суммирования ряда ${ }^{N} F$. Основное содержание принципа факторизации заключается в утверждении: если $D(i)=D_{1}(i) D_{2}(i)$, то ${ }^{N} F[D] \equiv{ }^{N} F\left[D_{1} \times D_{2}\right]={ }^{N} F\left[D_{1}\right] *{ }^{N} F\left[D_{2}\right]$ (свойство $\Omega$-представимости операции умножения коэффициентов степенного ряда). Будем назьвать такую операторную редукцию (связывающую ряды от одинакового числа переменных) общей редукиией или редукиией $(N, N)$-типа.

Работаподдержана Российским фондом фундаментальных исследований (грант № 97-01-00317). 
В случае общего гипергеометрического ряда ${ }^{N} F$ для каждого из сомножителей $D_{k}(\boldsymbol{i})$, входящих в произведение $D(\boldsymbol{i})$, выполняется специальное условие "спектральной представимости" $D_{k}(\boldsymbol{i})=L_{k}(\boldsymbol{m} \cdot \boldsymbol{i}), \boldsymbol{m} \cdot \boldsymbol{i} \equiv m_{1} i_{1}+\cdots+m_{N} i_{N}$, где $L_{k}(I)-$ некоторая функция от одного переменного, а $m_{1}, \ldots, m_{N}$ - произвольные целые числа, именуемые далее спектральными параметрами ряда ${ }^{N_{F}}$. Как показано в [2], в случае $m_{1} \geqslant 0, \ldots, m_{N} \geqslant 0$ для “спектрально представимого" ряда ${ }^{N} F$ наряду с общей редукцией $(N, N)$-типа имеет место специальная операторная редукция $(N, 1)$-типа, выражающая ряд ${ }^{N} F$ в виде $\Omega$-произведения более простого ряда от $N$ переменных и ряда ${ }^{1} F\left[L_{k}\right]$ от одного переменного. Специальные формулы факторизации играют важную роль в операторной теории гипергеометрических функций, так как позволяют осуществить “глобальную редукцию” ряда к гипергеометрическим функциям от одного переменного.

Условие неотрицательности спектральных параметров, использованное в [2] для доказательства специальных формул редукции, существенно ограничивает область применимости метода факторизации, так как многие типы гипергеометрических рядов (например, 19 из 34 функций Горна [4], большинство тройных рядов Шриваштавы и Карлссона [5], гипергеометрические функции Гельфанда, Граева и Ретаха [6]-[8] и т. д. содержат не только положительные, но и отрицательные спектральные индексы.

В настоящей заметке показано, что существование обобщенной операции $\Omega$-умножения, применимой к рядам с произвольны.ми цельми спектральными индексами и непротиворечашей первоначальному определению гипергеометрических рядов общего вида, в некотором смысле завершающее построение "операторной теории" гипергеометрических функций, не сопровождается усложнением технического аппарата метода, так как основные алгебраические свойства операции $\Omega$-умножения сохраняются при переходе к обобшенному случаю. Это позволяет применить для исследования сложных гипергеометрических функций простые и эффективные правила операторного исчисления, которые были применены ранее [1]-[3] для анализа функций болеепростого вида. В качестве иллюстрации этого утверждения в заметке дан вывод некоторых формул преобразования горновского ряда $G_{3}$, содержащего два индефинитных спектральных набора, и проведено сравнение полученных результатов с литературными данными, требующими применения значительно более сложных методов.

Прежде чем перейти к изложению основных результатов, введем удобные обозначения для общих гипергеометрических рядов и поясним некоторые свойства и определения, используемые в дальнейшем.

2. Общие гипергеометрические ряды: обозначения, определения, элементарные свойства. За исключением символа $\boldsymbol{d}$ все остальные "векторные" символы используем далее либо для обозначения $N$-компонентных наборов, связанных с переменными суммирования ряда ${ }^{N} F$, либо для обозначения числовых величин, связанных с такими наборами. Например, для обозначения наборов величин $i_{n}, x_{n}, m_{n}^{a}, l_{n}^{b}$, $d\left(s_{n}\right) \equiv d / d s_{n}, x_{n} s_{n}, x_{n} s^{m_{n}}, x_{n} u^{m_{n}},(-1)^{m_{n}} x_{n}$, для каждого из которых $n=1, \ldots, N$, будем использовать векторные символы $\boldsymbol{i}, \boldsymbol{x}, \boldsymbol{m}^{a}, \boldsymbol{l}^{b}, d(s), \boldsymbol{x} s, \boldsymbol{x} s^{\boldsymbol{m}}, \boldsymbol{x} u^{\boldsymbol{m}}$ и $(-1)^{\boldsymbol{m}} \boldsymbol{x}$ соответственно. Для обозначения "скалярных" величин, связанных с этими "векторами", применим символы: $\boldsymbol{m}^{a} \cdot \boldsymbol{i}=m_{1}^{a} i_{1}+\cdots+m_{N}^{a} i_{N}, \boldsymbol{l}^{b} \cdot \boldsymbol{i}=l_{1}^{b} i_{1}+\cdots+l_{N}^{b} i_{N}$, $\boldsymbol{m} \cdot \boldsymbol{v}=m_{1} v_{1}+\cdots+m_{N} v_{N}, \boldsymbol{x}^{\boldsymbol{i}}=\prod_{n=1}^{N} x_{n}^{i_{n}}, \boldsymbol{x}^{\boldsymbol{i}+\boldsymbol{v}}=\prod_{n=1}^{N} x_{n}^{i_{n}+v_{n}}, \boldsymbol{i} !=\prod_{n=1}^{N} i_{n} !$, $\Gamma(1+\boldsymbol{i}+\boldsymbol{v})=\prod_{n=1}^{N} \Gamma\left(1+i_{n}+v_{n}\right)$. Совокупности условий $0 \leqslant i_{n}<\infty,-\infty<i_{n}<\infty$, $v_{n} \rightarrow 0, m_{n} \geqslant 0$ и т. п., где $n=1, \ldots, N$, будем также записьвать в "векторной" форме: 
$0 \leqslant i<\infty,-\infty<i<\infty, \boldsymbol{v} \rightarrow 0, \boldsymbol{m} \geqslant 0$ и т. д.

ОПРедЕЛЕнИЕ 1. Общим гипергеометрическим рядом от $N$ переменньх, характеризуемым списком параметров $L$, назовем степенной ряд вида

$$
\begin{gathered}
{ }^{N} F[L ; \boldsymbol{x}] \equiv{ }^{N_{F}}\left[\begin{array}{c}
\left\langle\alpha^{1} \mid \boldsymbol{m}^{1}\right\rangle, \ldots,\left\langle\alpha^{A} \mid \boldsymbol{m}^{A}\right\rangle ; \boldsymbol{x} \\
\left\langle\beta^{1} \mid \boldsymbol{l}^{1}\right\rangle, \ldots,\left\langle\beta^{B} \mid \boldsymbol{l}^{B}\right\rangle
\end{array}\right]=\sum_{\boldsymbol{i}=0}^{\infty} L(\boldsymbol{i}) \frac{\boldsymbol{x}^{\boldsymbol{i}}}{\boldsymbol{i} !}, \\
L(\boldsymbol{i})=\left[\left(\alpha^{1}, \boldsymbol{m}^{1} \cdot \boldsymbol{i}\right) \ldots\left(\alpha^{A}, \boldsymbol{m}^{A} \cdot \boldsymbol{i}\right)\right] /\left[\left(\beta^{1}, \boldsymbol{l}^{1} \cdot \boldsymbol{i}\right) \ldots\left(\beta^{B}, \boldsymbol{l}^{B} \cdot \boldsymbol{i}\right)\right],
\end{gathered}
$$

где символы Похгаммера $(a, p)$, связанные с параметрами числителя $\alpha^{a}$ и параметрами знаменателя $\beta^{b}$, определяются соотношением $(a, p)=\Gamma(a+p) / \Gamma(a)$, а спектральные индексы $m_{1}^{a}, \ldots, m_{N}^{a}(1 \leqslant a \leqslant A)$ и $l_{1}^{b}, \ldots, l_{N}^{b}(1 \leqslant b \leqslant B)$ принимают произвольные целые значения, совместимые с условиями сходимости ряда ${ }^{N} F$.

ОПредЕЛЕниЕ 2. Параметры ряда ${ }^{N} F$, имеющие одинаковыеспектральные наборы, будем назьвать спектрально әквивалентными.

Для сокращения записи будем объединять такиепараметрыв әлементарные списки вида

$$
\left[\begin{array}{c}
\langle a \mid \boldsymbol{m}\rangle,\langle b \mid \boldsymbol{m}\rangle, \ldots \\
\langle c \mid \boldsymbol{m}\rangle,\langle e \mid \boldsymbol{m}\rangle, \ldots
\end{array}\right]=\left\langle\begin{array}{c}
a, b, \ldots \\
c, e, \ldots
\end{array} \mid \boldsymbol{m}\right\rangle=\langle\boldsymbol{d} \mid \boldsymbol{m}\rangle, \boldsymbol{d} \equiv\left[\begin{array}{c}
a, b, \ldots \\
c, e, \ldots
\end{array}\right]
$$

где $\boldsymbol{d}$ - произвольный двойной набор спектрально эквивалентных параметров, входящих в список $L$. В однострочных обозначениях будем отделять параметры числителя любого двойного набора от параметров его знаменателя двойной наклонной чертой, например, $\boldsymbol{d}=[a, b, \ldots] / /[c, e, \ldots]$ и т. п.

ОПрЕДЕЛЕниЕ 3. Если спектральньй набор $\boldsymbol{m}$, входящий в элементарньй список $\langle\boldsymbol{d} \mid \boldsymbol{m}\rangle$, имеет вид $\boldsymbol{m}=\left(0, \ldots, 0, m_{n}, 0, \ldots, 0\right)$, будем назьвать набор параметров $\boldsymbol{d}$ индивидуальным набором, связанным с $n$-м переменньм суммирования. В противном случае набор параметров $\boldsymbol{d}$ будем назьвать глюонным (склеивающим) набором.

Полный список параметров ряда ${ }^{N} F$ будем записывать в виде $L=\left[L(G): L_{1} ; \ldots ; L_{N}\right]$, где $L(G)$ - список глюонных параметров, а $\left[L_{1} ; \ldots ; L_{N}\right]-$ упорядоченньй набор индивидуальных списков, помеченных номерами соответствующих переменных суммирования ряда ${ }^{N} F$. Применение позиционных обозначений позволяет использовать вместо развернутой записи индивидуальных элементарных списков $\left\langle\boldsymbol{d} \mid 0, \ldots, m_{n}, \ldots, 0\right\rangle,\left\langle\boldsymbol{d}^{\prime}\right| 0$, $\left.\ldots, m_{n}^{\prime}, \ldots, 0\right\rangle$, входящих в объединенный индивидуальный список $L_{n}$, сокращенную запись вида $L_{n}=\left[\left\langle\boldsymbol{d} \mid m_{n}\right\rangle,\left\langle\boldsymbol{d}^{\prime} \mid m_{n}^{\prime}\right\rangle, \ldots\right]$.

ОПредЕЛЕниЕ 4. Глюонньй набор $\boldsymbol{d}$ или индивидуальный набор $\boldsymbol{d}^{\prime}$ будем назьвать простыми наборами, если все компоненты спектрального набора $\boldsymbol{m}$, входящего в глюонньй элементарньй список $\langle\boldsymbol{d} \mid \boldsymbol{m}\rangle$, или спектральное число $m_{n}$, входящее в индивидуальньй элементарный список $\left\langle\boldsymbol{d}^{\prime} \mid m_{n}\right\rangle$, имеют единичные значения.

Простые наборы $\boldsymbol{d}$ и $\boldsymbol{d}^{\prime}$ будем заносить в соответствующие объединенные списки $L(G)$ и $L_{n}$ без указания спектральных индексов. Другими словами, символы $\boldsymbol{d}$ и $\boldsymbol{d}^{\prime}$ в списках $L(G)$ и $L_{n}$ будем считать эквивалентными символам $\langle\boldsymbol{d} \mid 1,1, \ldots, 1\rangle$, и $\left\langle\boldsymbol{d}^{\prime} \mid 1\right\rangle$ соответственно.

Пустое множсество (или подмножество) параметров в любой части полного списка $L$ будем обозначать символом $*$, опуская этот значок (или множество таких значков) в тех случаях, ког да это не приводит к недоразумениям. Например, ${ }^{N} F[L(G): * ; * ; \ldots ; * ; \boldsymbol{x}]$ $\equiv{ }^{N} F[L(G) ; \boldsymbol{x}],{ }^{2} F\left[a / / b: a_{1} / / * ; a_{2} / / * ; x_{1}, x_{2}\right] \equiv{ }^{2} F\left[a / / b: a_{1} ; a_{2} ; x_{1}, x_{2}\right]$ и т. п. 
ЗАмЕчАниЕ 1. Для любого ряда ${ }^{N} F$ имеет место преобразование инверсии

$$
{ }^{N} F[\langle\alpha \mid \boldsymbol{m}\rangle, L ; \boldsymbol{x}]={ }^{N} F\left[L / /\langle 1-\alpha \mid \overline{\boldsymbol{m}}\rangle ;(-1)^{\boldsymbol{m}} \boldsymbol{x}\right], \quad \overline{\boldsymbol{m}} \equiv-\boldsymbol{m},
$$

котороепозволяет: (а) перевести любой параметр ряда из числителя в знаменатель или из знаменателя в числитель; (б) изменить знаки всех компонент любого спектрального набора на противоположные; (в) придать заданной компоненте любого спектрального набора неотрицательное значение; (г) привести к спектрально эквивалентной форме параметры, отличающиеся инверсией (изменением знаков всех компонент) спектра.

Справедливость формулы (3), а вместе с нею и замечания 1 следует из соотношений $(1),(2)$ с помощью элементарного тождества $(\alpha, i)=(-1)^{i} /(1-\alpha,-i)$. Замечание 1 а показывает, что разделение параметров ряда ${ }^{N} F$ на параметры числителя и параметры знаменателя имеет условньй характер. Следует, однако, иметь в виду, что разбиение множества параметров ряда на подмножества спектрально эквивалентных параметров (см. замечание 1г), значительно упрощающее структуру многих формул факторизации, в общем случае неизбежно сопровождается разделением параметров ряда на параметры числителя и параметры знаменателя.

ЗАмЕчАниЕ 2. Параметры, характеризующиеся кратными спектрами, могут быть приведены к спектрально эквивалентной форме с помощью формулы умножения Гаусса-Лежандра.

В самом деле, если ряд ${ }^{N} F$ содержит параметры $\langle\alpha \mid \boldsymbol{m}\rangle$ и $\langle\beta \mid \boldsymbol{l}\rangle$ такие,что $\boldsymbol{m}=k \boldsymbol{l}$, где $k$ - целое положительное число, то коэффициенты, соответствующие этим параметрам, имеют вид $(\alpha, k i)$ и $(\beta, i)$, где $i=\boldsymbol{l} \cdot \boldsymbol{i}$. В этом случае справедливость замечания 2 вытекает из формулы $(\alpha, k i)=k^{k i}(\alpha / k, i)((\alpha+1) / k, i) \ldots((\alpha+k-1) / k, i)$, являющейся парафразом формулы умножения Гаусса-Лежандра [4]. Если $k$ - целое отрицательное число, то с помощью замечания 16 доказательство замечания 2 сводится к рассмотренному вьше случаю.

Непосредственно из замечания 2 и определения 4 следует:

ЗАмЕчАниЕ 3. Параметры, спектры которых кратны единичному спектру, представимы в виде набора простых параметров. Любой набор индивидуальных параметров представим в виде набора простых индивидуальных параметров.

ЗАмечАниЕ 4. Гипергеометрический ряд ${ }^{N} F$ симметричен относительно любой перестановки аргументов ряда и одновременной перестановки соответствующих индивидуальных наборов и соответствующих компонент всех спектральных наборов.

Данное утверждение вытекает из определений 1 и 3.

ОПРЕДЕЛЕНИЕ 5. Парииальным типом ряда ${ }^{N} F$ (по некоторому переменному $x_{n}$ ) будем назьвать пару чисел $\left(p_{n} / / q_{n}\right)$, где

$$
\begin{gathered}
p_{n}=\frac{\sigma_{n}+\delta_{n}}{2}, \quad q_{n}=\frac{\sigma_{n}-\delta_{n}}{2}, \\
\sigma_{n}=\sum_{a=1}^{A}\left|m_{n}^{a}\right|+\sum_{b=1}^{B}\left|l_{n}^{b}\right|, \quad \delta_{n}=\sum_{a=1}^{A} m_{n}^{a}-\sum_{b=1}^{B} l_{n}^{b}, \quad n=1,2, \ldots, N .
\end{gathered}
$$




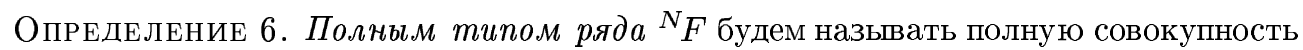
парциальных типов по всем переменным, т.е. таблицу чисел вида $\left(p_{1} / / q_{1}, \ldots, p_{N} / / q_{N}\right)$.

ОПРЕДЕЛЕНИЕ 7 . Наибольшее из чисел $p_{1}, \ldots, p_{N}, q_{1}+1, \ldots, q_{N}+1$ будем назьвать порядком ряда ${ }^{N} F$.

В случае $N=2$ определение 7 эквивалентно определению порядка, предложенному Горном [4]. В соответствии с терминологией, принятой в [4] для случая $N=2$, будем назьвать ряд ${ }^{N} F$ полным гипергеометрическим рядом, если $p_{1}=\cdots=p_{N}=q_{1}+1=$ $\cdots=q_{N}+1$. Классификация рядов по типам дает более содержательную информацию о структуре ряда ${ }^{N} F$ по сравнению с общепринятой классификацией по порядку.

3. Обобщенные формулы факторизации. Для ряда ${ }^{N} F$ имеет место общая формула факторизации $(N, N)$-типа [2]:

$$
{ }^{N} F\left[L_{1}, L_{2} ; \boldsymbol{x}\right]=\left.{ }^{N} F\left[L_{1} ; d(\boldsymbol{s})\right]^{N} F\left[L_{2} ; \boldsymbol{x s}\right]\right|_{\boldsymbol{s}=0},
$$

где $d(s)=\left[d\left(s_{1}\right), \ldots, d\left(s_{N}\right)\right], d\left(s_{n}\right)=d / d s_{n}$, a $\left[L_{1}, L_{2}\right]-$ произвольное разбиение списка $L$ на два списка меньшего объема. Применение формулы (4) не приводит к изменению структуры элементарных списков вида $\langle\boldsymbol{d} \mid \boldsymbol{m}\rangle$, входящих в список $L$. В случае $\boldsymbol{m} \geqslant 0$ возможна более глубокая перестройка ряда ${ }^{N} F$ с помощью специальной формулы факторизации $(N, 1)$-типа [3]:

$$
{ }^{N} F\left[\langle\boldsymbol{d} \mid \boldsymbol{m}\rangle, L ; \boldsymbol{x} u^{\boldsymbol{m}}\right]=\left.F[\boldsymbol{d} ; u d(s)]^{N} F\left[L ; \boldsymbol{x} s^{\boldsymbol{m}}\right]\right|_{s=0}
$$

где $\boldsymbol{F}[\boldsymbol{d} ; \boldsymbol{x}]$ - обобщенньй гипергеометрический ряд от одного переменного [4]

$$
F[\boldsymbol{d} ; x] \equiv F_{q}^{p}[\boldsymbol{d} ; x]=\sum_{i=0}^{\infty}(\boldsymbol{d}, i) \frac{x^{i}}{i !}=\sum_{i=0}^{\infty}\{[(a, i)(b, i) \ldots] /[(c, i)(e, i) \ldots]\} \frac{x^{i}}{i !}
$$

содержащий $p$ параметров числителя $a, b, \ldots$ и $q$ параметров знаменателя $c, e, \ldots$.

Для доказательства формул $(4),(5)$ достаточно использовать в их правых частях определения (1), (6) и применить затем основное дифференииальное тождество $\left.d^{i}(s) s^{j}\right|_{s=0}=i ! \delta(i, j)(i=0,1,2, \ldots ; j=0,1,2, \ldots)$, где $\delta(i, j)$ - символ Кронекера, принимаюший значения 1 и 0 в случаях $i=j$ и $i \neq j$ соответственно. В случае формулы (5) имеем $j=\boldsymbol{m} \cdot \boldsymbol{i}$, где $\boldsymbol{i}$ - набор переменных суммирования ряда ${ }^{N} F[L]$. Поскольку основное дифференциальное тождество имеет место только в том случае, если $i \geqslant 0$ и $j \geqslant 0$, то приведенное доказательство соотношения (5) применимо лишь для $\boldsymbol{m} \geqslant 0$ (или для $\boldsymbol{m} \leqslant 0$, см. замечание 1б).

Чтобы распространить формулу (5) на случай индефинитных спектральных наборов, сопоставим степенньм рядам тейлоровского типа (6) и (1) формальные степенные ряды лорановского типа:

$$
F_{v}[\boldsymbol{d} ; x]=\sum_{i=-\infty}^{\infty} \frac{(\boldsymbol{d}, i+v) x^{i+v}}{\Gamma(1+i+v)}, \quad{ }^{N} F_{\boldsymbol{v}}[L ; \boldsymbol{x}]=\sum_{\boldsymbol{i}=-\infty}^{\infty} \frac{L(\boldsymbol{i}+\boldsymbol{v}) \boldsymbol{x}^{\boldsymbol{i}+\boldsymbol{v}}}{\Gamma(1+\boldsymbol{i}+\boldsymbol{v})}
$$


где $v, v_{1}, \ldots, v_{N}$ - произвольные вещественные числа. Почленное применение обобщенной формулы дифференцирования [9] $d^{\nu}(s) s^{\mu}=[\Gamma(1+\mu) / \Gamma(1+\mu-\nu)] s^{\mu-\nu}$ позволяет определить $\Omega$-произведение рядов (7а) и (7б) в виде:

$$
\Omega_{\boldsymbol{v}}^{v}(\boldsymbol{m} ; \boldsymbol{x})=\left.F_{v}[\boldsymbol{d} ; d(s)]^{N} F_{\boldsymbol{v}}\left[L ; \boldsymbol{x} s^{\boldsymbol{m}}\right]\right|_{s=0} .
$$

В общем случае предел последовательности функций (8) при $v \rightarrow 0, \boldsymbol{v} \rightarrow 0$ не существует, однакоглавное значение этого предела, соответствующее дополнительному условию $v=\boldsymbol{m} \cdot \boldsymbol{v}$, сушествует, а его явное выражение для любых целых $m_{1}, \ldots, m_{N}$ имеет вид ряда ${ }^{N} F[\langle\boldsymbol{d} \mid \boldsymbol{m}\rangle, L ; \boldsymbol{x}]$, совпадающего по форме с левой частью (5). Другими словами, имеет место

ПРЕДЛОЖЕНИЕ 1. Обобщенная формула факторизаиии ряда ${ }^{N} F[\langle\boldsymbol{d} \mid \boldsymbol{m}\rangle, L ; \boldsymbol{x}]$ имеem вид

$$
{ }^{N} F[\langle\boldsymbol{d} \mid \boldsymbol{m}\rangle, L ; \boldsymbol{x}]=\left.\lim _{\boldsymbol{v} \rightarrow 0} F_{\boldsymbol{m} \cdot \boldsymbol{v}}[\boldsymbol{d} ; d(s)]^{N} F_{\boldsymbol{v}}\left[L ; \boldsymbol{x} s^{\boldsymbol{m}}\right]\right|_{s=0},
$$

где $m_{1}, \ldots, m_{N}-$ произвольные целые числа.

ДоКАЗАТЕЛЬСТВО. Подставляя определения (7а) и (7б) в правую часть (8) и применяя почленно обобщенную формулу дифференцирования, получаем:

$$
\Omega_{\boldsymbol{v}}^{v}=\left.\sum_{i=-\infty}^{\infty} \sum_{i=-\infty}^{\infty} \frac{(\boldsymbol{d}, i+v) L(\boldsymbol{i}+\boldsymbol{v}) \boldsymbol{x}^{i+\boldsymbol{v}} \Gamma\left(1+i^{\prime}+v^{\prime}\right) s^{i^{\prime}-i+v^{\prime}-v}}{\Gamma(1+i+v) \Gamma(1+\boldsymbol{i}+\boldsymbol{v}) \Gamma\left(1+i^{\prime}-i+v^{\prime}-v\right)}\right|_{s=0},
$$

где $v^{\prime} \equiv \boldsymbol{m} \cdot \boldsymbol{v}, i^{\prime} \equiv \boldsymbol{m} \cdot \boldsymbol{i}$. Поскольку функция $\Gamma(z)$ имеет полюсы в точках $z=0,-1,-2$, $\ldots$, общий член ряда (10) в случае $v=v^{\prime}$ отличен от нуля, если $i^{\prime}-i \geqslant 0$. При выполнение условий $i^{\prime} \geqslant i, v=v^{\prime}$ предел величины $s^{i^{\prime}-i+v^{\prime}-v}=s^{i^{\prime}-i}$ при $s \rightarrow 0$ существует и равен $\delta\left(i, i^{\prime}\right)$. После вьполнения предельного перехода при $s \rightarrow 0$ видно, что $(N+1)$-кратная сумма в правой части (10) в силу “правила отбора" $i=i^{\prime}$ сводится к $N$-кратной сумме по переменньм $i_{1}, \ldots, i_{N}$, а отношение $\Gamma\left(1+i^{\prime}+v^{\prime}\right) / \Gamma(1+i+v)$ обращается в единицу. Переходя затем к пределу при $v \rightarrow 0$ и учитьвая, что $1 / \Gamma(1+i) \neq 0$ в том и только том случае, если $i \geqslant 0$, получаем $\Omega_{0, \ldots, 0}^{0}(\boldsymbol{m} ; \boldsymbol{x})={ }^{N} F[\langle\boldsymbol{d} \mid \boldsymbol{m}\rangle, L ; \boldsymbol{x}]$, что завершает доказательство предложения 1.

Таким образом, несмотря на то, что в случае индефинитных спектральных наборов операция $\Omega$-умножения не может быть определена непосредственно на множестве рядов тейлоровского типа, ее можно определить на более широком множестве степенных рядов лорановского типа. Главное значениепредела последовательности $\Omega$-произведений, соответствующего переходу к граничным (тейлоровским) элементам этого множества, существует и может быть принято за определение обобщенного $\Omega$-произведения.

Алгебраические свойства операции $\Omega$-умножения [1] определяются коммутационными свойствами операторов $d(s)$ и $s$. Поскольку операции дифференцирования и предельного перехода в правой части (9) независимы друг от друга, имеет место

ПРЕДЛОЖЕНИЕ 2. Наличие предельного перехода $v \rightarrow 0$ в правой части (9), отличающее формулу (9) от соотношения (5), не приводит к изменению алгебраических свойств операции обобщенного $\Omega$-умножсения по сравнению с операчией обичного $\Omega$-умножения.

В частности, для операции обобщенного $\Omega$-умножения сохраняются свойства коммутативности и ассоциативности, правило спаривания, свойство $\Omega$ - единичности показательной функции и т. п. (см. [1]-[3]). Это позволяет отказаться от введения специальньх 
обозначений для обобщенного $\Omega$-произведения, т. е. сохранить для этого случая обычные дифференциальные обозначения типа (5), не прибегая к явному указанию предельных переходов, а лишь подразумевая их в необходимьх случаях.

4. Трансформационные свойства горновского ряда $G_{3}$. В теории гипергеометрических рядов важную роль играют формулы преобразования вида $F=\widetilde{F}$, выражающие некоторый ряд $F$ в виде другого ряда (или комбинации некоторых рядов) $\widetilde{F}$. Для вывода таких формул обычно используют один из трех методов: метод прямой перегрупшировки ряда $F$; метод, основанный на применении дифференциальных соотношений для функций $F$ и $\widetilde{F}$; и метод интегральных преобразований [5]. Гипергеометрические ряды с индефинитными спектральными наборами наиболее трудны для изучения. Для исследования трансформационных свойств горновских рядов с индефинитными спектрами Эрдейи предложил использовать метод контурных интегралов по двойной петле Похгаммера [10]. В качестве примера, иллюстрирующего эффективность метода контурных интегралов, Шриваштава и Карлссон (см. [5, с. 318]) приводят соотношение Эрдейи [10]

$$
\begin{gathered}
G_{3}\left[\alpha_{1}, \alpha_{2} ; y_{1} X_{2} X_{1}^{-2}, y_{2} X_{1} X_{2}^{-2}\right] \\
=X_{1}^{\alpha_{2}} X_{2}^{\alpha_{1}} F_{2}\left[1-\alpha, \alpha, \alpha, 1-\alpha_{1}, 1-\alpha_{2} ; x_{1}, x_{2}\right] \\
X_{1}=1-y_{1}, \quad X_{2}=1-y_{2}, \quad y_{1}=x_{1}\left(1-x_{2}\right), \\
y_{2}=x_{2}\left(1-x_{1}\right), \quad \alpha \equiv \alpha_{1}+\alpha_{2},
\end{gathered}
$$

выражающее горновскую функцию $G_{3}$ через функцию Аппеля $F_{2}$, и указьвают, ссылаясь на замечание Эрдейи [10], что это преобразование "было бы трудно получить с помощью любого из первых двух (упомянутых выще) методов”. Однако, легко показать, что соотношение (11), по крайней мере в той форме, в какой оно приведено в [5], является ошибочньм. В самом деле, применяя обозначения, введенные в п. 2, запишем определения функций $G_{3}$ и $F_{2}$ в виде $G_{3}\left[\alpha_{1}, \alpha_{2} ; x_{1}, x_{2}\right]={ }^{2} F\left[\left\langle\alpha_{1} \mid \overline{1}, 2\right\rangle,\left\langle\alpha_{2} \mid 2, \overline{1}\right\rangle ; x_{1}, x_{2}\right]$, $F_{2}\left[\alpha, a_{1}, a_{2}, b_{1}, b_{2} ; x_{1}, x_{2}\right]={ }^{2} F\left[\alpha: a_{1} / / b_{1} ; a_{2} / / b_{2} ; x_{1}, x_{2}\right]$. В случае $x_{2}=0$ с учетом этих определений и замечаний 1,2 и 3 из (11) следует, что

$$
F_{1}^{2}\left[\frac{\alpha_{2}}{2}, \frac{\alpha_{2}+1}{2} / / 1-\alpha_{1} ; \frac{-4 x_{1}}{\left(1-x_{1}\right)^{2}}\right]=\left(1-x_{1}\right)^{\alpha_{2}} F_{1}^{2}\left[1-\alpha_{12}, \alpha_{12} / / 1-\alpha_{1} ; x_{1}\right]
$$

где $\alpha_{12} \equiv \alpha_{1}+\alpha_{2}$. Чтобы убедиться в некорректности формулы (12), достаточно положить в ней $\alpha_{1}=\left(1-\alpha_{2}\right) / 2$, что приводит к явно ошибочному соотношению $F_{0}^{1}\left[\alpha_{2} / 2\right.$; $\left.-4 x_{1}\left(1-x_{1}\right)^{-2}\right]=\left(1-x_{1}\right)^{\alpha_{2}} F_{0}^{1}\left[\left(1-\alpha_{2}\right) / 2 ; x_{1}\right]$, где $F_{0}^{1}[a ; x]=(1-x)^{-a}$.

Применяя по каждому из параметров ряда $G_{3}$ обобщенную формулу факторизации (5) (см. предложения 1 и 2) и учитывая вспомогательные операторные тождества [2], [3]

$$
\begin{aligned}
& \left.F(d(s)) \Psi\left(x z^{m} s^{m}\right)\right|_{s=0}=\left.F(z d(s)) \Psi\left(x s^{m}\right)\right|_{s=0}, \\
& \left.\quad F_{0}^{1}[\alpha ; z d(s)] \Psi(s)\right|_{s=0}=\left.z^{-\alpha} F_{0}^{1}[\alpha ; d(s)] \exp \left[\left(1-z^{-1}\right) s\right] \Psi(s)\right|_{s=0},
\end{aligned}
$$

где $F, \Psi$ - произвольные функции, а $F_{0}^{1}[\alpha ; z]=(1-z)^{-\alpha}$, имеем

$$
\begin{aligned}
G_{3} & {\left[\alpha_{1}, \alpha_{2} ; x_{1} z_{1}^{-1} z_{2}^{2}, x_{2} z_{1}^{2} z_{2}^{-1}\right] \equiv{ }^{2} F\left[\left\langle\alpha_{1} \mid \overline{1}, 2\right\rangle,\left\langle\alpha_{2} \mid 2, \overline{1}\right\rangle ; x_{1} z_{1}^{-1} z_{2}^{2}, x_{2} z_{1}^{2} z_{2}^{-1}\right] } \\
& =\left.F_{0}^{1}\left[\alpha_{1} ; z_{1} d\left(s_{1}\right)\right] F_{0}^{1}\left[\alpha_{2} ; z_{2} d\left(s_{2}\right)\right] \exp \left(x_{1} s_{1}^{-1} s_{2}^{2}+x_{2} s_{1}^{2} s_{2}^{-1}\right)\right|_{s_{1}=s_{2}=0} \\
& =\left.z_{1}^{-\alpha_{1}} z^{-\alpha_{2}} F_{0}^{1}\left[\alpha_{1} ; d\left(s_{1}\right)\right] F_{0}^{1}\left[\alpha_{2} ; d\left(s_{2}\right)\right] E\left(s_{1}, s_{2}\right)\right|_{s_{1}=s_{2}=0}
\end{aligned}
$$


где $E\left(s_{1}, s_{2}\right)=\exp \left[Q\left(s_{1}, s_{2}\right) / s_{1} s_{2}\right]$, а $Q\left(s_{1}, s_{2}\right)$ - кубическая форма вида

$$
Q\left(s_{1}, s_{2}\right)=x_{2} s_{1}^{3}+\left(1-z_{1}^{-1}\right) s_{1}^{2} s_{2}+\left(1-z_{2}^{-1}\right) s_{1} s_{2}^{2}+x_{1} s_{2}^{3} .
$$

В двух случаях $n=1,2$, соответствующих специальным значениям параметров $z_{i}=$ $z_{i, n}=\left(1+x_{i}-n x_{1}-n x_{2}\right)^{-1}$, где $i=1,2$, кубическая форма $Q$ принимает особо простые выражения вида $Q=Q_{n}=\left(s_{1}+s_{2}\right)^{n}\left(x_{2} s_{1}^{3-n}+x_{1} s_{2}^{3-n}\right)$. Применение этих выражений в формуле для $E$ позволяет применить к функции $E\left(s_{1}, s_{2}\right)$ формулу сложения $\exp (a+b)=\exp (a) \exp (b)$. Записывая каждую из экспонент $\exp (a)$ и $\exp (b)$ в виде степенного ряда, после подстановки преобразованной $E$ в (13) получаем:

$$
\begin{gathered}
G_{3}=z_{1, n}^{-\alpha_{1}} z_{2, n}^{-\alpha_{2}} \sum_{i_{1}, i_{2}} L\left(i_{1}, i_{2}\right) \frac{x_{1}^{i_{1}} x_{2}^{i_{2}}}{i_{1} ! i_{2} !} \\
L\left(i_{1}, i_{2}\right)=\left.F_{0}^{1}\left[\alpha_{1} ; d\left(s_{1}\right)\right] s_{1}^{\nu_{1}} F_{0}^{1}\left[\alpha_{2} ; d\left(s_{2}\right)\right] s_{2}^{\nu_{2}}\left(s_{1}+s_{2}\right)^{i}\right|_{s_{1}=s_{2}=0} \\
\nu_{1} \equiv(2-n) i_{2}-i_{1}, \quad \nu_{2} \equiv(2-n) i_{1}-i_{2}, \quad i \equiv n\left(i_{1}+i_{2}\right) .
\end{gathered}
$$

Используя в правой части (16) свойство коммутативности операции $\Omega$-умножения [1], учитьвая правило дифференцирования $d^{\nu}(s) F_{0}^{1}[\alpha ; s]=(\alpha, \nu) F_{0}^{1}[\alpha+\nu ; s]$ и применяя затем обобщенное правило Лейбница [1], имеем:

$$
L\left(i_{1}, i_{2}\right)=\left.\left(\alpha_{1}, \nu_{1}\right)\left(\alpha_{2}, \nu_{2}\right) d^{i}(s) F_{0}^{1}\left[\alpha_{1}+\nu_{1} ; s\right] F_{0}^{1}\left[\alpha_{2}+\nu_{2} ; s\right]\right|_{s=0} .
$$

Применяя в правой части (18) формулу сложения $F_{0}^{1} F_{0}^{1}=F_{0}^{1}\left[\alpha_{12}+\nu_{12} ; s\right]$, где $\alpha_{12} \equiv$ $\alpha_{1}+\alpha_{2}, \nu_{12} \equiv \nu_{1}+\nu_{2}$, и учитьвая, что $\left.d^{i}(s) F\left[\alpha_{12}+\nu_{12} ; s\right]\right|_{s=0}=\left(\alpha_{12}+\nu_{12}, i\right)$, после несложных преобразований получаем

$$
L\left(i_{1}, i_{2}\right)=\sigma_{n}^{i_{12}}\left(\alpha_{1}, \nu_{1}\right)\left(\alpha_{2}, \nu_{2}\right)\left(\alpha_{12}, i_{12}\right)\left(1-\alpha_{12},(n-1) i_{12}\right),
$$

где $\sigma_{n}=(-1)^{n-1}, i_{12}=i_{1}+i_{2}$. Подставляя (19) в (15), учитывая определения (17), (1) и применяя обозначения, введенные в п. 2 , имеем:

$$
\begin{aligned}
G_{3}= & z_{1, n}^{-\alpha_{1}} z_{2, n}^{-\alpha_{2}} \\
& \times{ }^{2} F\left[\alpha_{12},\left\langle 1-\alpha_{12} \mid n-1, n-1\right\rangle,\left\langle\alpha_{1} \mid \overline{1}, 2-n\right\rangle,\left\langle\alpha_{2} \mid 2-n, \overline{1}\right\rangle ; \sigma_{n} x_{1}, \sigma_{n} x_{2}\right]
\end{aligned}
$$

Рассматривая в (20) по-отдельности случаи $n=1$ и $n=2$, используя явные выражения для параметров $z_{1, n}$ и $z_{2, n}$, применяя в случае $n=2$ замечания $1 \mathrm{a}, 1$ в и учитьвая определения горновских функций $G_{1}$ и $F_{4}[4],[5]$, получаем окончательную формулировку результата:

ПРЕДЛОЖЕНИЕ 3. Для

$$
\begin{aligned}
& G_{3}\left[\alpha_{1}, \alpha_{2} ; \frac{x_{1}\left(1-x_{2}\right)}{\left(1-x_{1}\right)^{2}}, \frac{x_{2}\left(1-x_{1}\right)}{\left(1-x_{2}\right)^{2}}\right] \\
& \quad=\left(1-x_{2}\right)^{\alpha_{1}}\left(1-x_{1}\right)^{\alpha_{2}} G_{1}\left[\alpha_{12}, \alpha_{1}, \alpha_{2} ; x_{1}, x_{2}\right] \\
& G_{3}\left[\alpha_{1}, \alpha_{2} ; \frac{x_{1}\left(1-x_{1}-2 x_{2}\right)}{\left(1-x_{2}-2 x_{1}\right)^{2}}, \frac{x_{2}\left(1-x_{2}-2 x_{1}\right)}{\left(1-x_{1}-2 x_{2}\right)^{2}}\right] \\
& \quad=\left(1-x_{1}-2 x_{2}\right)^{\alpha_{1}}\left(1-x_{2}-2 x_{1}\right)^{\alpha_{2}} F_{4}\left[\alpha_{12}, 1-\alpha_{12}, 1-\alpha_{1}, 1-\alpha_{2} ; x_{1}, x_{2}\right]
\end{aligned}
$$


связывающие этот ряд с горновской функиией $G_{1}\left[\alpha, \alpha_{1}, \alpha_{2}\right] \equiv{ }^{2} F\left[\alpha,\left\langle\alpha_{1} \mid \overline{1}, 1\right\rangle,\left\langle\alpha_{2} \mid 1, \overline{1}\right\rangle\right]$ и функиией Аппеля $F_{4}\left[a_{1}, a_{2}, b_{1}, b_{2}\right] \equiv{ }^{2} F\left[a_{1}, a_{2}: * / / b_{1} ; * / / b_{2}\right]$.

Формулы (21), (22) позволяют найти корректньй аналог ошибочной формулы (11). Например, применяя в правой части (22) формулу Бейли [11] (см. также [5, с. 318]) $F_{4}\left[a, b_{1}+b_{2}-1, b_{1}, b_{2} ; z_{1}\left(1-z_{2}\right), z_{2}\left(1-z_{1}\right)\right]=F_{2}\left[b_{1}+b_{2}-1, a, a, b_{1}, b_{2} ; z_{1}, z_{2}\right]$, легко показать, что преобразование (11) имеет место в том случае, если

$$
\begin{gathered}
X_{1}=1-y_{2}-2 y_{1}, \quad X_{2}=1-y_{1}-2 y_{2}, \\
y_{1}=x_{1}\left(1-x_{2}\right), \quad y_{2}=x_{2}\left(1-x_{1}\right), \quad \alpha \equiv \alpha_{1}+\alpha_{2} .
\end{gathered}
$$

Для дополнительной проверки формул (23) можно применить в правой части (21) формулу Эрдейи [12], связывающую функцию $G_{1}$ с $F_{2}$ (см. также [5, c. 319]).

Отметим в заключение, что существует еще один специальньй случай $z_{i}=1 /[1-$ $\left.3\left(x_{1} x_{2} x_{i}\right)^{1 / 3}\right]$, для которого кубическая форма $Q$ (см. (14)) принимает особо простой вид $Q=\left(x_{2}^{1 / 3} s_{1}+x_{1}^{1 / 3} s_{2}\right)^{3}$. По аналогии с выводом формул $(21),(22)$ можно показать, что ряд $G_{3}$ допускает в этом случае кубические преобразования, связывающие его с негорновскими рядами типов $(6 / / 5,2 / / 1),(5 / / 4,2 / / 1),(4 / / 3,2 / / 1)$ и $(3 / / 2,2 / / 1)$.

\section{СПИСОК ЦИТИРОВАННОЙ ЛИТЕРАТУРЫ}

[1] Niukkanen A. W. Generalized operator reduction formulae for multiple hypergeometric series ${ }^{N} F\left(x_{1}, \ldots, x_{N}\right) / /$ J. Phys. A. Math. Gen. 1984. V. 17. P. 731-736.

[2] Ниукканен А. В. Новый метод в теории гипергеометрических рядов и специальных функций математической физики // Успехи матем. наук. 1988. Т. 43. № 3. С. 191-192.

[3] Ниукканен А. В. Новый подход к теории гипергеометрических рядов и специальных функций математической физики // Матем. заметки. 1991. Т. 50. № 1. С. 65-73.

[4] Бейтмен Г., Эрдейи А. Высшие трансцендентные функции. Т. 1. М.: Наука, 1973.

[5] Srivastava H. M., Karlsson P.W. Multiple Hypergeometric Series. Chichester: Ellis Hoorwood, 1985.

[6] Гельфанд И.М., Граев М.И., Ретах В.С. Г-ряды и общие гипергеометрические функции на многообразии $(k \times n)$-матриц. Препринт ИПМ, 1990 .

[7] Гельфанд И. М., Граев М. И. Гипергеометрические функции, связанные с грассманианом $G_{3,6} / /$ Матем. сб. 1989. Т. 180. № 1. С. 3-38.

[8] Гельфанд И.М., Граев М.И., Ретах В.С. Общие гипергеометрические системы уравнений и ряды гипергеометрического типа // Успехи матем. наук. 1992. Т. 47. № 4. С. 3-82.

[9] Гельфанд И. М., Шилов Г. Е. Обобщенные функции и действия над ними. М: Физматгиз, 1958.

[10] Erdélyi A. Transformations of hypergeometric functions of two variables // Proc. Roy. Soc. Edinburg Sect. A. 1948. V. 62. P. 378-385.

[11] Bailey W. N. Generalized hypergeometric series. Cambridge: Cambridge University Press, 1935.

[12] Erdélyi A. Hypergeometric functions of two variables // Acta Math. 1950. V. 83. P. 131-164. 